\title{
Aplicação de perfis geofísicos em projetos de perfuração de poços de petróleo no Golfo de México
}

\author{
Marcus Vinicius Leite ${ }^{1}$, Antonio Abel Carrasquilla ${ }^{2}$,Jadir Conceição da Silva ${ }^{3}$ (in memoriam), \\ Luiz Lobo ${ }^{1}$, Rafael Curtinhas ${ }^{1}$ \& Sandro Batista ${ }^{4}$
}

\begin{abstract}
Resumo A elaboração de um projeto de perfuração de poços de petróleo pode ser realizada através de uma abordagem que envolva conhecimentos científicos de várias disciplinas, tais como geologia, geofísica e engenharia de petróleo. Por este motivo, é de grande importância atuar na interface dessas áreas, a fim de mostrar como essa interação pode proporcionar uma visão interdisciplinar da fenomenologia envolvida no processo. No presente trabalho, foram analisados dados mecânicos, geológicos e geofísicos de dois poços do Golfo de México, com a finalidade de conhecer os parâmetros de perfuração e selecionar as brocas, fazendo possível, assim, a montagem de uma estratégia de perfuração de outros poços no campo estudado. Os resultados alcançados com o estudo dos perfis geofísicos indicam que, nos três intervalos observados, melhores brocas poderiam ter sido selecionadas. Entretanto, é importante ressaltar a experiência dos profissionais envolvidos num projeto de perfuração, pois, diversas vezes é preferível perfurar mais devagar e evitar a troca de broca por desgaste, que furar de forma rápida, mas ter que trocar continuamente a broca. Por fim, a utilização integrada de dados dos poços permite a otimização do projeto e a elaboração de um plano de perfuração, o que se traduz na melhor utilização dos recursos econômicos locados nessa atividade.
\end{abstract}

Palavras-chave: perfis geofísicos de poço, Golfo do México, parâmetros de perfuração, estratégia de perfuração.

\begin{abstract}
Application of geophysical well logs on drilling exploration projects in the Gulf of Mexico. The execution of a drilling project of oil wells can be performed through an approach that involves scientific knowledge of diverse disciplines, such as geology, geophysicist and petroleum engineering. For this reason, it is of great importance to act in the interface of these areas, in order to demonstrate as this interaction can provide an interdisciplinary vision of the phenomena involved in the process. In the present work, mechanical, geological and geophysical data of two wells of the Gulf of Mexico had been analyzed, with the purpose to know the drilling parameters and to select the best perforation bits, making possible, thus, the assembly of a strategy of perforation of other wells in the studied oil field. The results reached in our study with the use of geophysical well logs indicate that, in the three observed intervals, better bits could have been selected. But, it is important to stand out that always the experience of the involved professionals in a drilling project must be considered. Therefore, several times is preferable to perforate slowly and to prevent the exchange of the consumed bits, that to drill in a fast form and have to change the bit continuously. However, the integrated use of the well data allows the optimization of the project and the elaboration of a perforation plan, which it is expressed in a better way to utilize the financial resources in this activity.
\end{abstract}

Keywords: geophysical well logging, Gulf of Mexico, drilling parameters, drilling strategy.

INTRODUÇÃ̃O Durante a elaboração de um projeto de perfuração são analisados os poços anteriormente perfurados no campo onde se planeja realizar uma nova locação. Nestas perfurações, denominadas de poços de correlação, são analisados a litologia, os perfis geofísicos de poço, os desgastes sofridos pelas brocas, as eventuais falhas ocorridas nos equipamentos, assim como, a compressibilidade uniaxial da rocha $\left(C^{\prime}\right)$. Com o cálculo de $C^{\prime}$ é possível determinar parâmetros a serem utilizados nesse processo, como peso sobre a broca $(P S B)$ e a rotação da coluna de perfuração (rpm), podendo-se, assim, traçar uma estratégia de perfuração através da escolha das brocas mais adequadas e dos equipamentos de perfuração, como por exemplo, motores de fundo (rotary steerable). Trabalhos como os de Farrelly \& Rabia (1987), Dumans (1990), Curry et al. (2005) e Dupriest \& Koederitz (2005) mostram a importância do conhecimento de $C^{\prime}$ da rocha para seleção de brocas, através do conceito de energia específica $\left(E_{\mathrm{s}}\right)$.

Neste trabalho, utilizamos dados de poços perfurados no Golfo do México (Caicedo et al. 2005), a fim de calcular $C^{\prime}$ em alguns intervalos perfurados e

1 -PETROBRAS, E\&P-SERV/US-PO, Imboassica, Macaé (RJ), Brasil. E-mail: marcosviniciuscl@gmail.com, rafael.curtinhas@petrobras.com.br

2 - Universidade Estadual do Norte Fluminense Darcy Ribeiro (LENEP/UENF), Laboratório de Engenharia e Exploração de Petróleo, Imboacica, Macaé (RJ), Brasil. E-mail: abel@lenep.uenf.br

3 - Universidade Federal do Rio de Janeiro, Departamento de Geologia, Ilha do Fundao, Rio de Janeiro (RJ), Brasil.

4 - Baker Hughes do Brasil Ltda, Balneario Lagomar, Macaé (RJ), Brasil. 
demonstrar como uma estratégia de perfuração pode ser montada mesmo sem a presença dos poços de correlação, ou seja, utilizando somente perfis geofísicos de poço, mas contemplando outras informações mecânicas e geológicas como forma de aferir os resultados. É importante ressaltar que essa abordagem é de natureza multidisciplinar, onde são usados conceitos de geologia, geofísica e engenharia de petróleo, buscando otimizar a elaboração de um projeto de perfuração.

CONTEXTO GEOLÓGICO O Golfo de México é um mar do tipo Mediterrâneo localizado na porção Sudeste da América do Norte, medindo, aproximadamente, $900 \mathrm{~km}$ de Norte a Sul e $1.600 \mathrm{~km}$ de Leste a Oeste, com uma superfície de 1, 5 milhões de $\mathrm{km}^{2}$ (Fig. 1). A bacia é composta por áreas rasas com menos de $20 \mathrm{~m}$ de profundidade (38\%), com uma plataforma $(<180 \mathrm{~m}$ de lâmina de água) e um talude $(180-3.000 \mathrm{~m})$ representando $22 \%$ e $20 \%$, respectivamente (Fig. 2). A sua planície abissal, com mais de $3,000 \mathrm{~m}$, compreende $20 \%$ da área total. Na sua parte mais funda, é preenchida com 10 a $15 \mathrm{~km}$ de rochas sedimentares com idades que vão do Triássico ao Holoceno (aproximadamente $230 \mathrm{Ma}$ ), sem evidências de rochas mais antigas (Fig. 3). Para compreender melhor a sua estrutura, deve-se considerar a história geológica de toda a Região do Caribe (175 Ma). A migração subseqüente de Gondwana para o Sul resultou numa grande conexão entre os oceanos Atlân- tico e Pacífico, que continuou a crescer até aproximadamente 100 Ma. Essa conexão foi parcialmente interrompida por dois deslocamentos expressivos de massa: primeiramente, as ilhas de Cuba e da Hispaniola foram originadas no Oceano Pacífico, na margem Oriental do Caribe; posteriormente, outros fragmentos continentais da mesma natureza surgiram ao Oeste, criando a América Central. O conjunto destes fechamentos gerou o Golfo de México e as bacias marginais do Caribe, o que alterou o fluxo original entre os oceanos e estabeleceu os padrões que prevalecem até hoje nessa região. Atualmente, entretanto, o Golfo de México é considerado geologicamente inativo, contrastando com as regiões do Caribe e da América Central, as quais estão sob uma forte influência da tectônica de placas (Gore 1992). Assim, desde o Jurássico, a bacia tem sido uma província geológica estável, caracterizada por uma subsidência constante na sua parte central, provavelmente devido ao resfriamento e a carga sedimentar que ela tem experimentado nas suas margens Norte e Noroeste, particularmente durante o Cenozoico (Fig. 4).

MATERIAIS E MÉTODOS O cálculo de $C^{\prime}$ pode ser realizado a partir da análise conjunta dos perfis de raios gama $(R G)$, sônico $(D T)$ e densidade $(r)$ dos poços de correlação, que são aqueles perfurados anteriormente na área de uma nova locação (Caicedo et al. 2005). Inicialmente, com o perfil $R G$, calcula-se o índice de

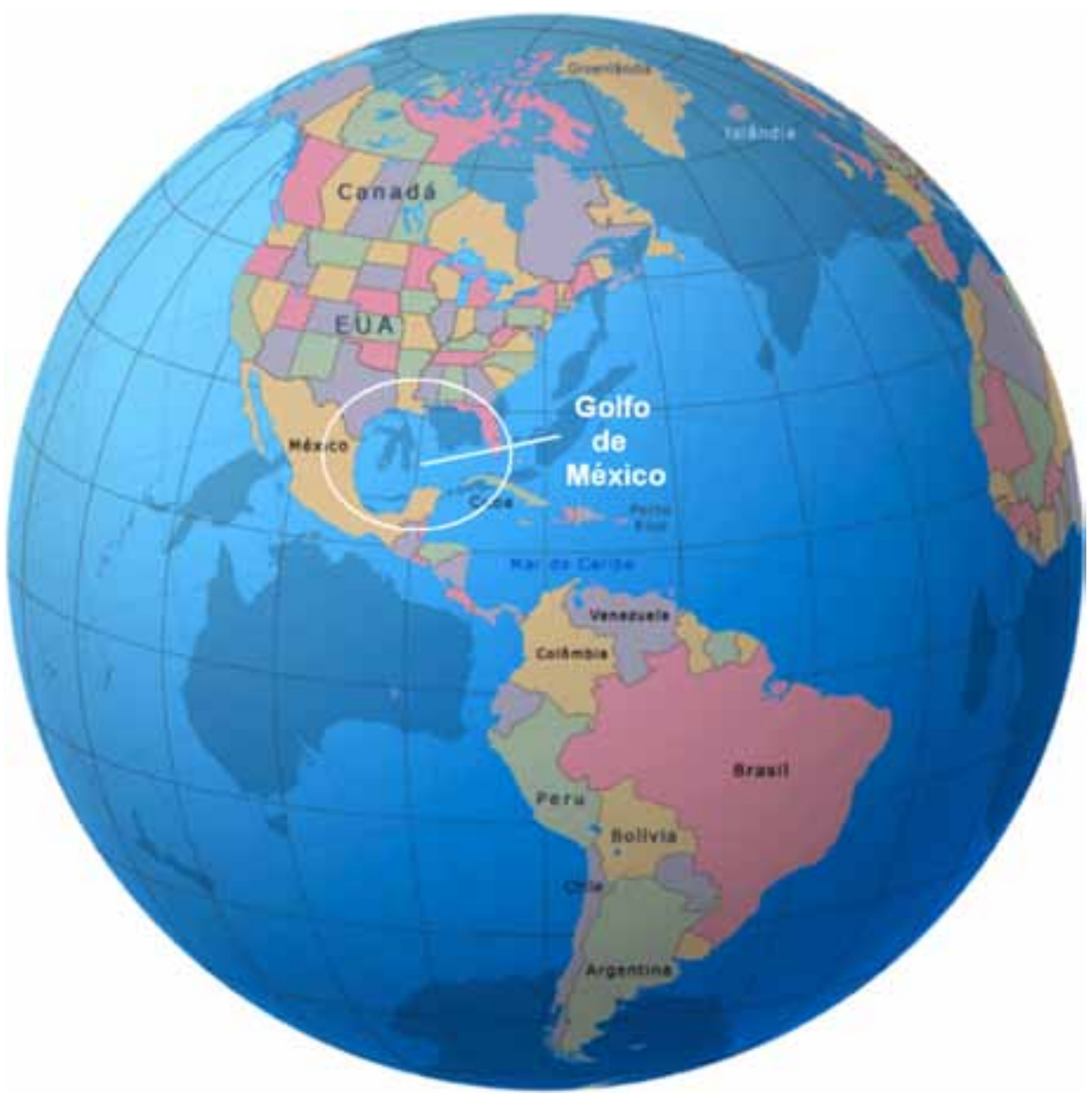

Figura 1 - Localização do Golfo de México (modificado de Gore 1992). 


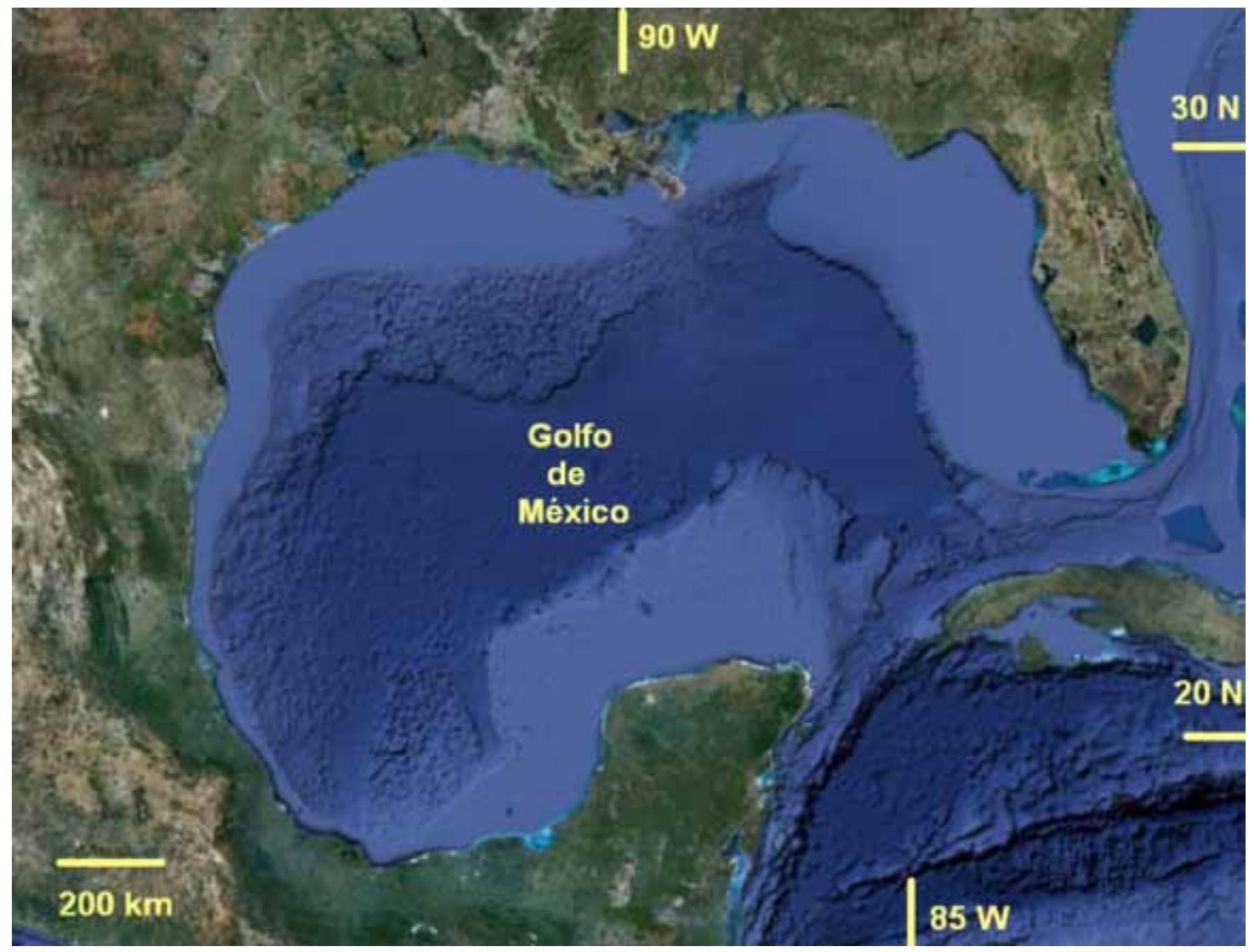

Figura 2 - Golfo de México em detalhes (modificado de Gore 1992).

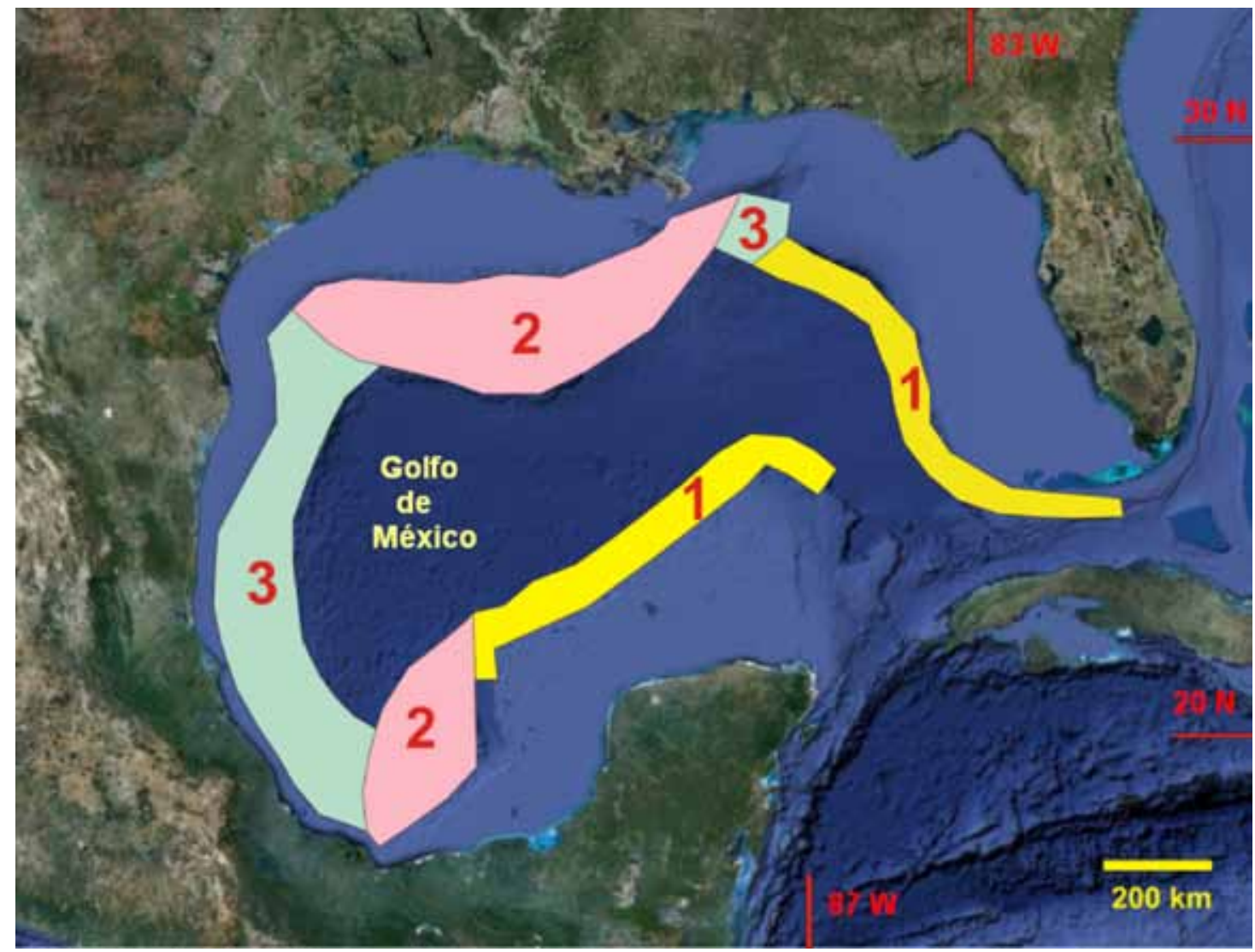

Figura 3 - O Golfo do México pode ser dividido em três áreas principais: escarpas profundas carbonáticas (tipo 1); camadas espessas de sedimentos com tectônica salina (tipo 2); e fundos oceânicos normais (tipo 3) (modificado de Gore 1992); 


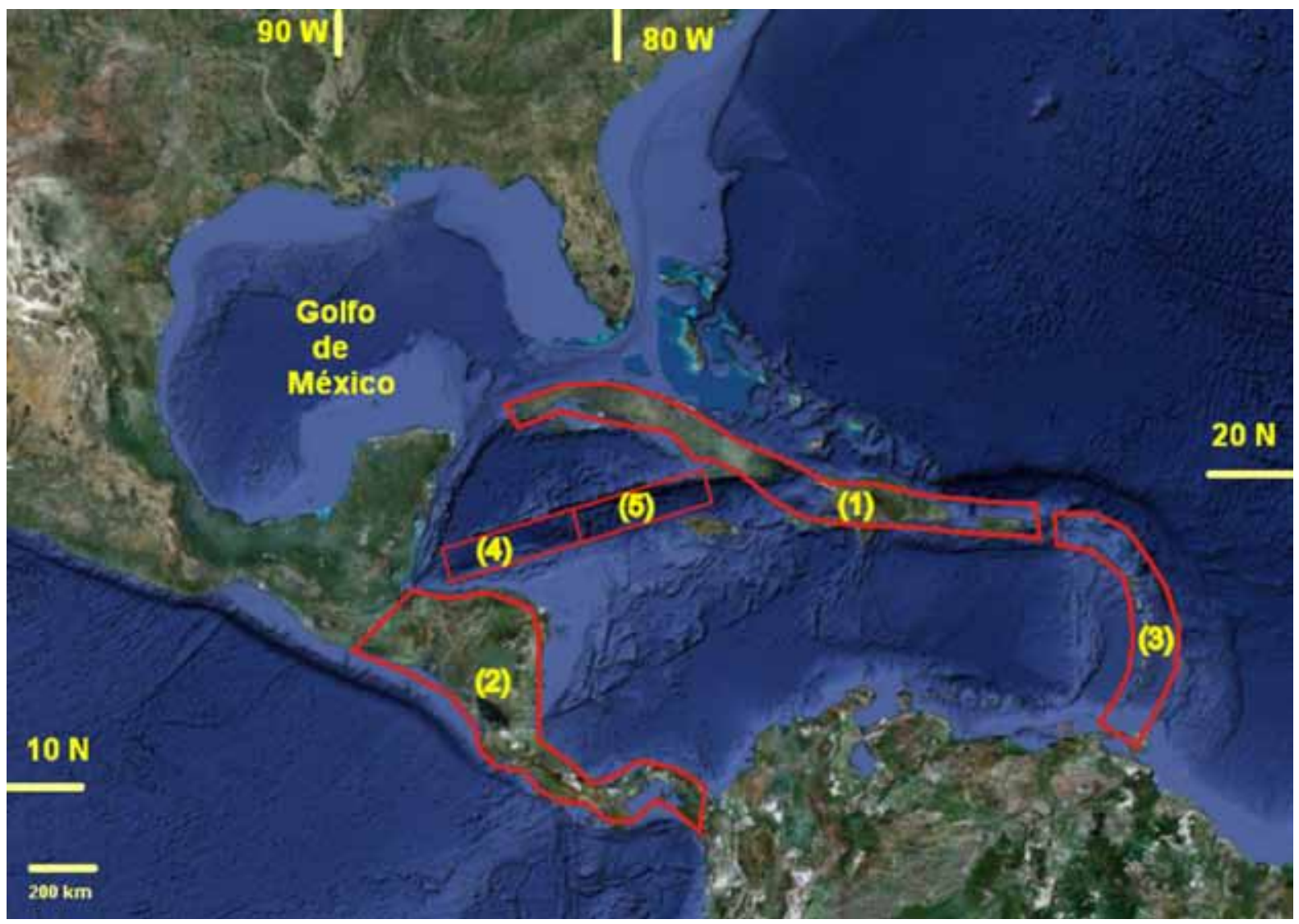

Figura 4 - Enquanto o Golfo de México é considerado geologicamente passivo, a região ao Sul é muito ativa, contando com estruturas que dão uma indicação da história geológica: fragmentos continentais antigos (1) e modernos (2), que fecharam o contato entre os dois oceanos ao Oeste; arco vulcânico ao Leste (3) e alinhamentos profundos (4) e (5), os quais são bons indicadores da contínua atividade no fundo do mar (modificado de Gore 1992).

raios gama $\left(I_{R G}\right)$ através da seguinte equação 1 (Asquith \& Gibson 1982):

$$
I_{R G}=\frac{R G_{\log }-R G_{\min }}{R G_{\max }-R G_{\min }}
$$

em que $R G_{l o g}$ é o valor do perfil $R G$ lido na formação; $R G_{\text {min }}$ é o valor mínimo do perfil $R G$ (areia limpa); e $R G_{\text {Max }}$ é o valor máximo do perfil $R G$ (folhelho). Por outro lado, o volume percentual de argila $\left(V_{s h}\right)$ é calculado através das equações 2 e 3 :

$$
V_{s h}=0,083\left\lfloor 2^{\left(3,7 I_{R G}\right)}-1\right\rfloor
$$

e,

$$
V_{s h}=0,33\left[2^{\left(2 I_{R G}\right)}-1\right.
$$

Rabelo (2004) apresentou as duas equações anteriores baseadas na idade da rocha, a primeira para rochas jovens do Terciário e a segunda para rochas mais antigas.

Conhecendo-se esses valores, é possível deri- var o valor de $C^{\prime}$ através da seguinte equação:

$C^{\prime}=3,3 X 10^{-20} \rho^{2} V_{p}^{4}\left(\frac{1+v}{1-v}\right)^{2}(1-2 v)\left(1+0,78 V_{s h}\right)$

em que $C^{\prime}$ é expressa em Mega Pascal (Mpa), $r$ é a massa específica da rocha obtida com o perfil de densidade $\left(\mathrm{kg} / \mathrm{m}^{3}\right) ; V$ é a velocidade da onda compressional derivada do perfil $D T(\mathrm{~m} / \mathrm{s}) ; V_{s h}$ é calculada através das equações 2 ou 3 com o auxilio do perfil $R G$, e n, é o módulo de Poisson, o qual é assumido com um valor médio de 0,25 (Erling \& Rune 1991).

Por outro lado, uma das formas de analisar o desempenho das brocas é através do conceito de $E_{S}$, o qual é definido como a quantidade de energia necessária para perfurar uma unidade de volume de uma determinada litologia, através da seguinte equação (Curry et al. 2005):

$$
E_{S}=\frac{P S B}{A_{B}}+\frac{120 \pi N T}{A_{B} T_{X}}
$$

em que $E_{s}$ é dado em libra por polegada quadrada (psi); $P S B$ é o peso sobre a broca em libra-força (lbf); $A_{B}$ é a área da broca (polegadas ${ }^{2}$ ); $N$ é a rotação na broca em 
revoluções por minuto (rpm); $T$ é o torque (lbf/pés); e $T_{x}$ é a taxa de penetração (pé/hora) (Dupriest \& Koederitz 2005). Teale (1965) introduziu os conceitos de energia específica mínima $\left(E_{\text {smin }}\right)$ e máxima $\left(E_{\text {smax }}\right)$, considerando que $E_{\text {smin }}=C^{\prime}$ na rocha perfurada e $E_{\text {smax }}$ será aquela atingida com os máximos valores de $P S B$ e $N$. Por conseguinte, com o conhecimento dos valores de $E_{s}, P S B, A_{B}$ e $T$ e utilizando esses conceitos, é possível estimar $C^{\prime}$, para, posteriormente, calcular $T_{x}$ através da equação 6 (Leite \& Lobo 2006):

$$
T_{X}=\frac{120 \pi N T}{E_{S} A_{B} P S B}
$$

Desta forma, a melhor broca a ser utilizada será aquela que apresentar maior $T_{x}$, ou seja, aquela que perfurar o intervalo em menor tempo, implicando em menores custos (Dagrain \& Tshibangu 2002).

RESULTADOS Para atingir os objetivos da nossa proposta no cálculo de $T_{x}$, o qual é baseado, por sua vez, na análise de perfis geofísicos de poços e nos conceitos de $E$, foram utilizados dados do Golfo do México através de dois poços denominados, neste artigo, de Poços 1 e 2.

Do Poço 1 foram analisados os intervalos de 4.614 a $4.768 \mathrm{~m}$ e 4.768 a $4.846 \mathrm{~m}$ da fase $81 / 2$ polegadas. O primeiro intervalo foi perfurado por uma broca tricônica 617 de insertos (Fig. 5), e o segundo intervalo com uma broca de PDC (Pollycrystaline Diamond Compact), conhecida como M1213 (Fig. 6), a qual encerra 9 lâminas e cortadores de $13 \mathrm{~mm}$ de diâmetro.

A nomenclatura utilizada na denominação das brocas tem o seguinte significado: o primeiro algarismo indica que a broca tem corpo de matriz, os dois seguintes o número de lâminas e os dois últimos o diâmetro em $\mathrm{mm}$. Por outro lado, a litologia perfurada neste intervalo foi calcilutito com $C^{\prime}$ mínima de 13.000 psi, média de 16.000 psi e máxima de 25.000 psi. No intervalo 4.614 a $4.768 \mathrm{~m}$ foi calculada a $T_{x}$ para brocas de dentes de aço 117, tricônica 517 e de inserto 447. Estes resultados foram comparados com a $T_{x}$ alcançada com a broca 617 , que realmente perfurou o intervalo (Fig. 7), enquanto a broca que obteve melhor desempenho foi a 117, com o segundo melhor desempenho para a 447. Nessa mesma figura é possível observar que $C^{\prime}$ é maior nos intervalos 4.640 - 4.650, 4.660 - 4.670 e 4.680 - 4.700, com valores de até $2,5 \times 10^{4}$ psi. Quase nesses mesmos intervalos mencionados, o valor da $P S B$ atinge valores máximos de $5,0 \times 10^{4} \mathrm{lb}$, com $N$ atingindo valores altos (»100 rpm) ao redor de $4.620 \mathrm{~m}$, e valores de quase $70,0 \mathrm{rpm}$ nos intervalos $4.660-4.680,4.710-4.725$ e acima de 4.740 $\mathrm{m}$. Os valores de $T$ são maiores, ao redor dos $4.620 \mathrm{~m}$ $(» 2,0 \mathrm{~m} / \mathrm{h})$, em $4.710 \mathrm{~m}(» 2,5 \mathrm{~m} / \mathrm{h})$ e $4.740 \mathrm{~m}(» 2,0 \mathrm{~m} / \mathrm{h})$. No caso de se perfurar poços no mesmo campo, estas seriam as duas melhores opções de brocas. É importante, também, considerar que a broca 117 se desgasta mais rápido que a 617 numa formação rochosa dura como a do Poço 1 , o que pode significar que a broca utilizada pode ter sido uma escolha certa por parte do engenhei-

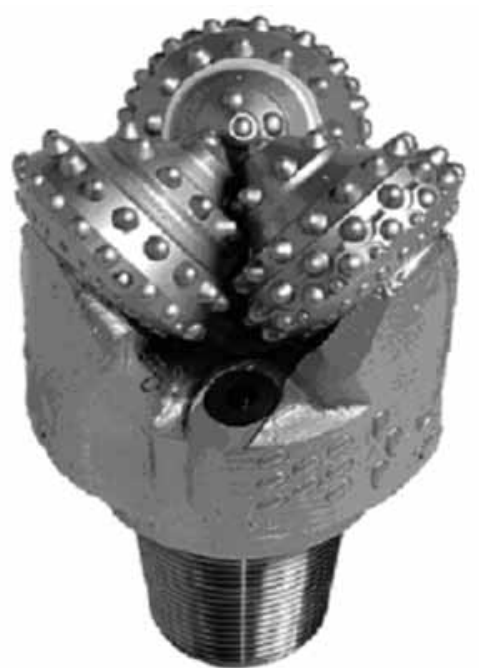

Figura 5 - Broca 8 1/2" tricônica de insertos 617 foi utilizada no intervalo de 4.614 a 4.768 do Poço 1 (modificada de www.bakerhughes. com, acesso em 15/01/2008).

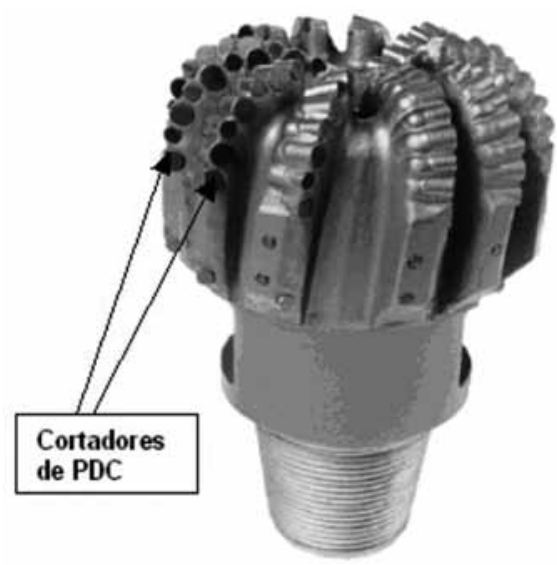

Figura 6 - Broca de PDC M1213, fabricada pela Smith Bits. Esta broca foi utilizada no Poço 1 para perfurar o intervalo de 4.768 a $4.846 \mathrm{~m}$ da fase de 8 1/2" (modificada de www.smithbits.com, acesso em 15/01/2008).

ro de perfuração, pois o desgaste é menor, evitando sua substituição, o que significaria retirar toda a coluna de perfuração para efetuar a troca.

No intervalo de $4.768 \mathrm{~m}$ a $4.846 \mathrm{~m}$ (Fig. 8), foram comparados os resultados de $T_{x}$ das brocas PDC M0516, M0719, M0916 e M1213. Esses valores são maiores, ao redor de $4.785 \mathrm{~m}(» 2,0 \mathrm{~m} / \mathrm{h}), 4.815 \mathrm{~m}(» 2,5$ $\mathrm{m} / \mathrm{h})$ e $4.845 \mathrm{~m}(» 2,0 \mathrm{~m} / \mathrm{h})$. Na mesma figura, é possível observar que $C^{\prime}$ é maior nos intervalos $4.780-4.800 \mathrm{~m}$ e acima de $4.820 \mathrm{~m}$, com valores de $1,8 \times 10^{4}$ e quase $2,0 \times 10^{4} \mathrm{psi}$, respectivamente. Entretanto, o valor da PSB é maior, ao redor dos $4.770 \mathrm{~m}$ com valores máximos de $4,0 \times 10^{4} \mathrm{lb}$ e acima de $4.840 \mathrm{~m}$, com valores de $4,0 \times 10^{4}$ 

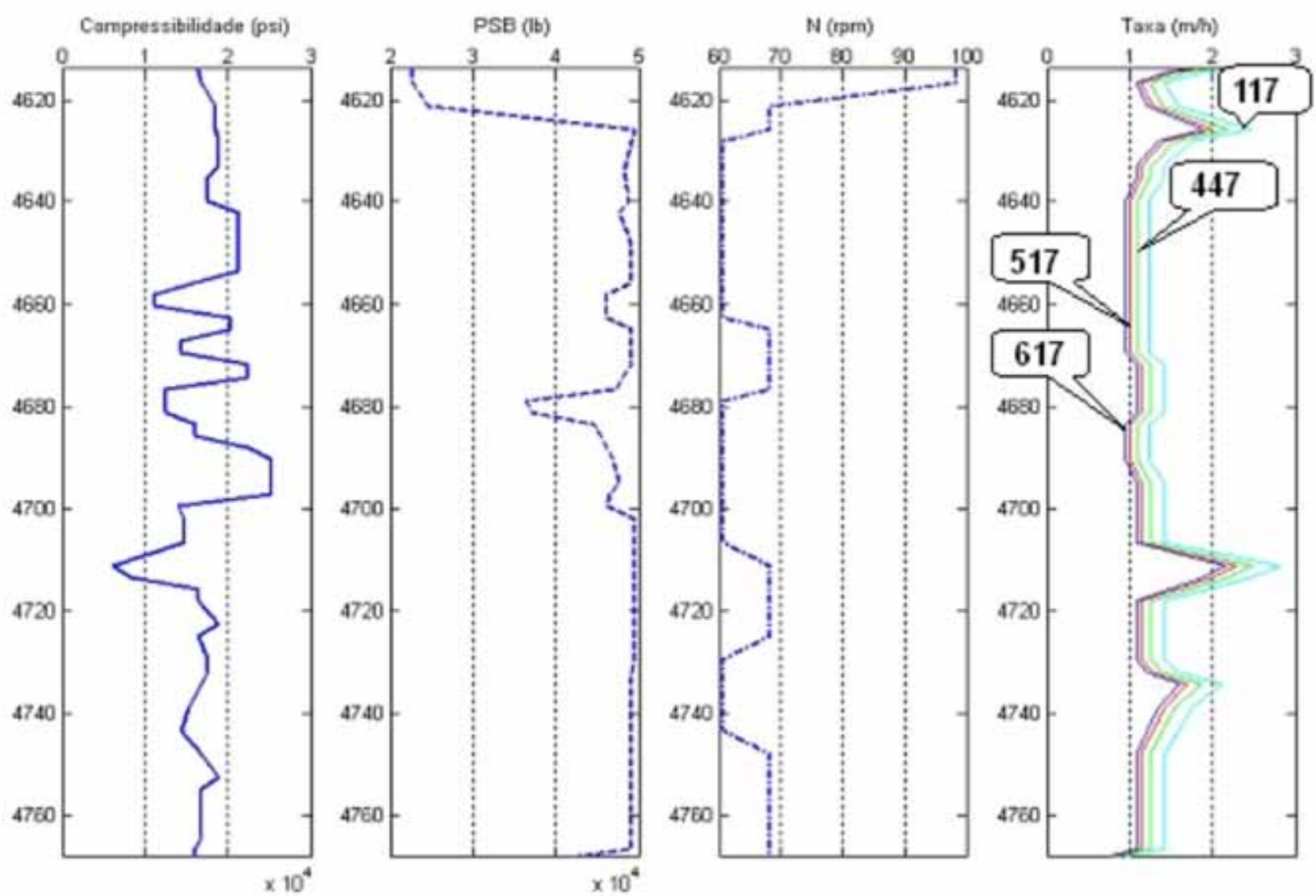

Figura 7 - Dados de litologia (calcilutito), compressibilidade (C'), peso sobre a broca (PSB), $N($ rpm) e taxa de penetração (T) do Poço 1. Na pista 4 pode-se observar as taxas de penetração previstas para os 4 tipos de brocas triconicas (117, 447, 517 e 617).
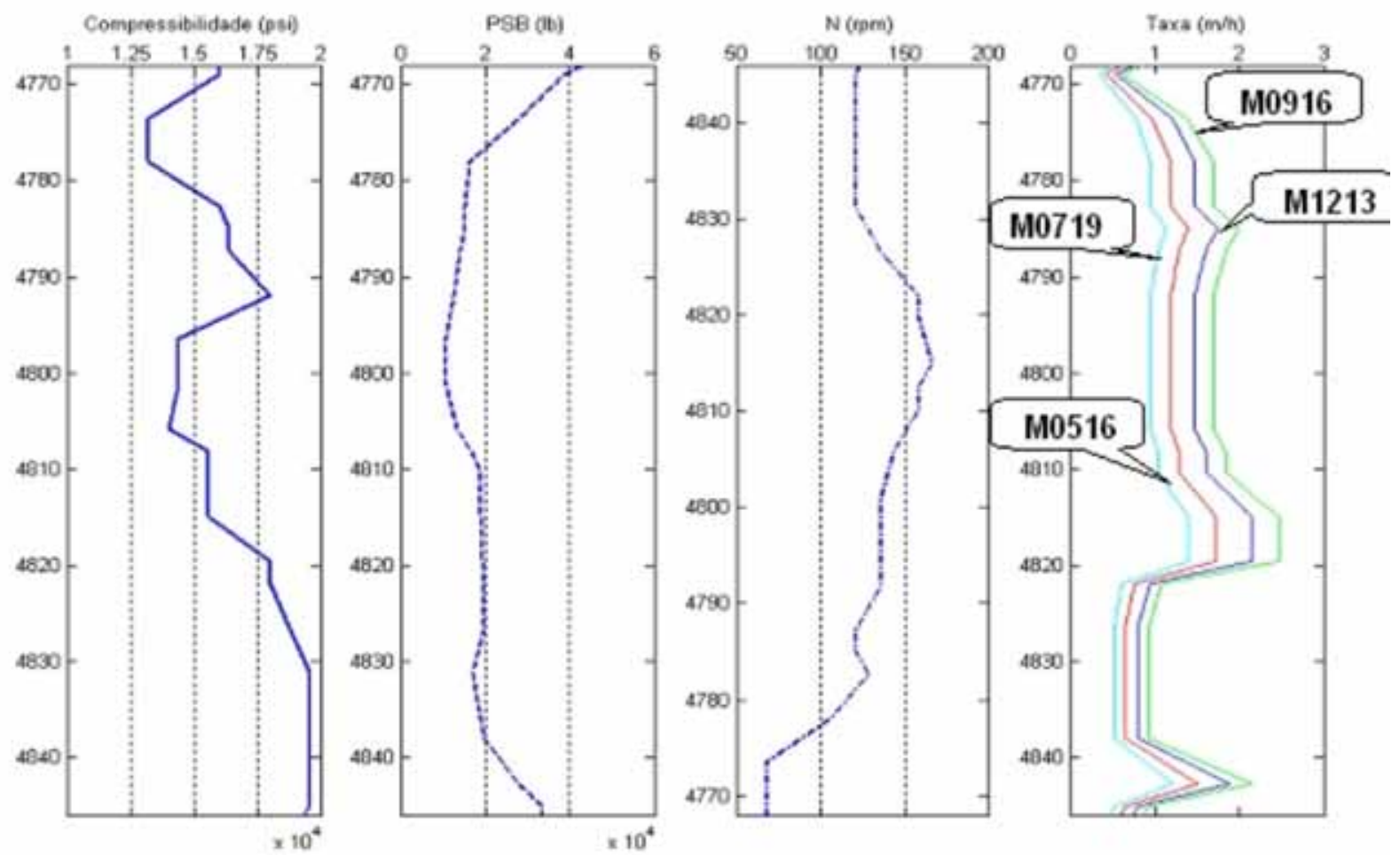

Figura 8 - Dados de litologia (folhelho com intercalações de arenito), compressibilidade ( $\left.C^{\prime}\right)$, peso sobre a broca (PSB), N e taxa de penetração (T) do Poço 2. Na pista 2 pode-se observar a taxa de penetração prevista para os 4 tipos de brocas de PDC (M0516, M0719, M0916 e M1213). 
lb. Por outro lado, $N$ atinge valores máximos (» $150 \mathrm{rpm}$ ) ao redor de $4820 \mathrm{~m}$, com valores baixos de quase $120 \mathrm{e}$ $70 \mathrm{rpm}$ nos intervalos maiores que $4.830 \mathrm{~m}$ e abaixo de $4.770 \mathrm{~m}$. Como pode ser observado nessa figura, a melhor opção para se perfurar outro intervalo semelhante num poço próximo a esta locação seria a broca M0916, cujos resultados mostram que ela seria superior a que foi utilizada na realidade, ou seja, a broca M1213.

Do Poço 2 foi analisado o intervalo de 7.315 $\mathrm{m}$ a $7.571 \mathrm{~m}$, composto por arenitos e folhelhos intercalados com $C^{\prime}$ mínima de 3.000 psi, média de 5.000 psi e máxima de 8.000 psi. A broca utilizada foi à PDC M0719 (Fig. 9). Neste caso, no intervalo de $7.315 \mathrm{~m}$ a $7.571 \mathrm{~m}$, a $T_{x}$ alcançada foi comparada a $T_{x}$ calculada para as brocas de PDC M0516, M0916 e M1213, sugerindo que a broca mais indicada para perfurar um próximo poço seria a M0916 (Fig. 10). Nessa figura, se observa que os maiores valores de $C^{\prime}$ estão ao redor das profundidades dos pontos $7.370 \mathrm{~m}(\gg 8.000 \mathrm{psi}), 7.430$ $\mathrm{m}$ (»7.000 psi), $7.470 \mathrm{~m}$ (»8.000 psi) e $7.560 \mathrm{~m}$ (acima de $8.000 \mathrm{psi}$ ). Em relação à $T$, ao redor das profundidades $7.400 \mathrm{~m}, 7.430 \mathrm{~m}$ e $7.550 \mathrm{~m}$, os valores são de até $40,0 \mathrm{~m} / \mathrm{h}$, enquanto nas alturas de $7.380 \mathrm{~m}$ e $7.480 \mathrm{~m}$, os valores são acima dos $40,0 \mathrm{~m} / \mathrm{h}$.

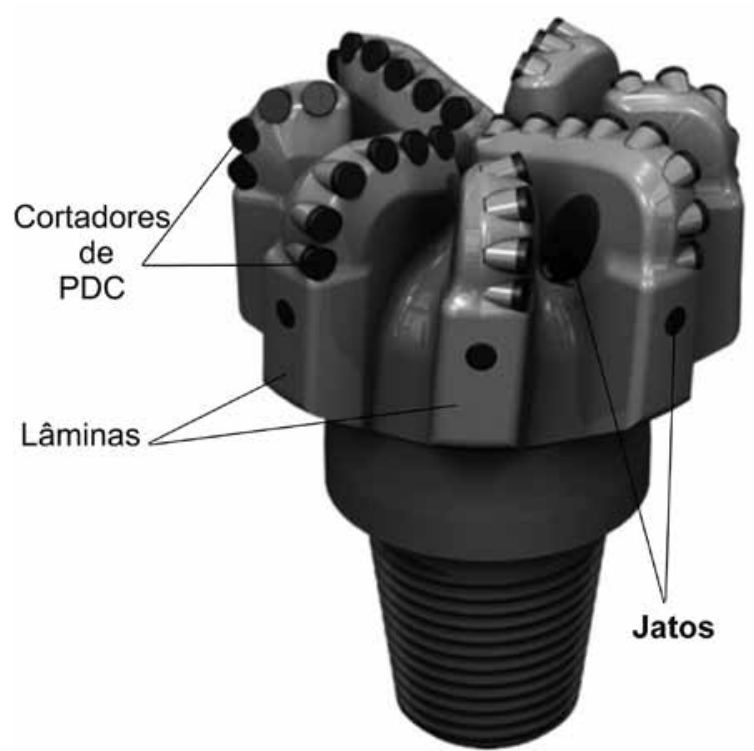

Figura 9 - Broca PDC M0719, fabricada pela Baker Hughes. A broca foi utilizada no intervalo de $7.315 \mathrm{~m}$ a $7.571 \mathrm{~m}$ da fase de $81 / 2$ " do Poço 2 (modificada de www.bakerhughes.com, acesso em 15/01/2008).
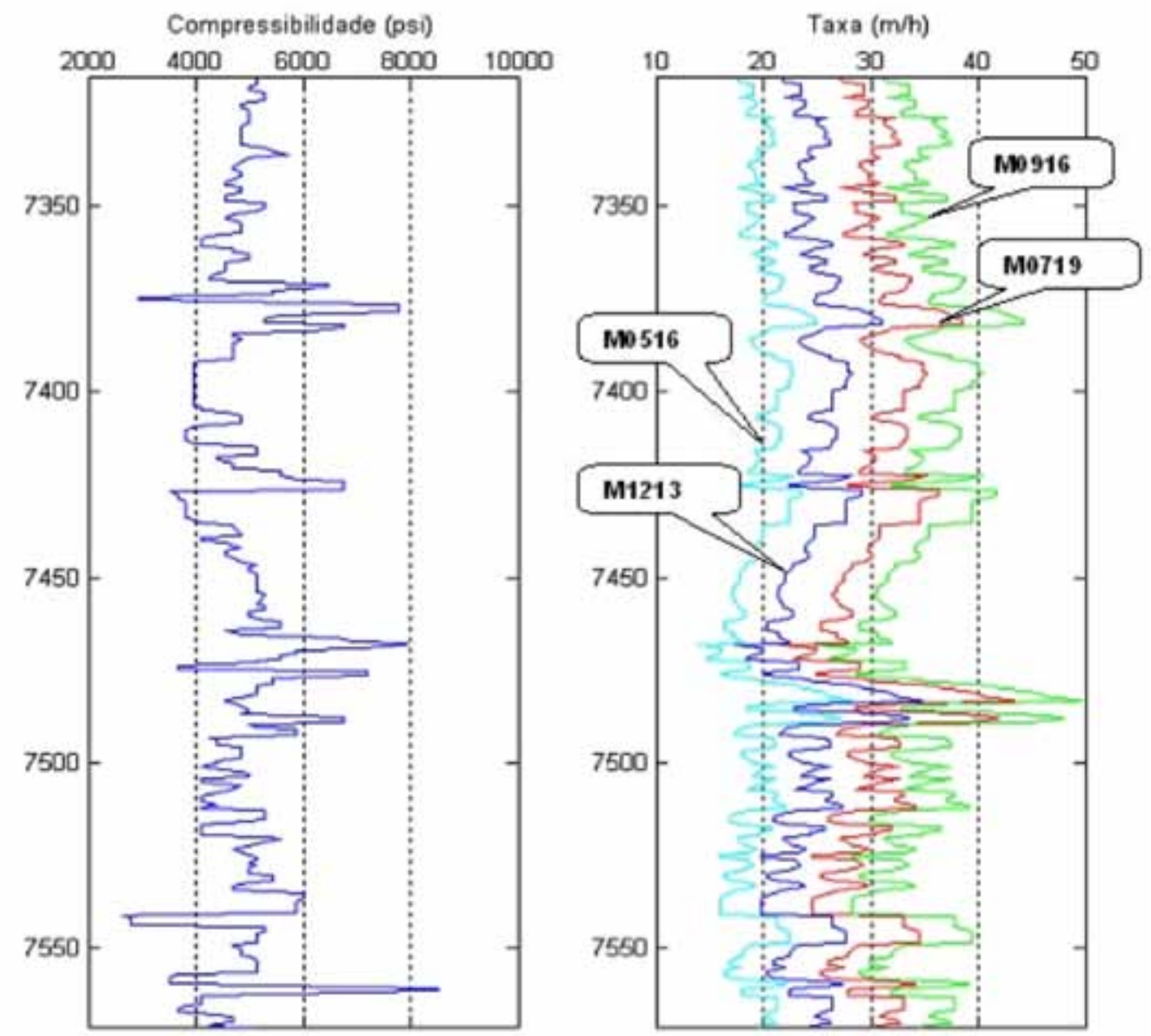

Figura 10 - Dados de litologia (folhelho com intercalações de arenito), compressibilidade $\left(C^{\prime}\right)$, peso sobre a broca (PSB), $N$ e taxa de penetração (T) do Poço 2. Na pista 2 pode-se observar a $T$ prevista para os 4 tipos de brocas de PDC (M0516, M0719, M0916 e M1213). 
Através da observação dos valores de $T_{x}$ e $C^{\prime}$ para ambos os poços estudados, é possível tirar conclusões sobre a dureza das litologias atravessadas, pois percebe-se que a do Poço 1 é maior que a do Poço 2, uma vez que perfurar com uma $T_{x}$ de no máximo 2,5 $\mathrm{m} / \mathrm{h}$ resulta numa $C^{\prime}$ muito pequena, com valores de no máximo 2,5 psi, enquanto no outro caso $T_{x}$ alcança valores maiores que $40,0 \mathrm{~m} / \mathrm{h}$ e $C^{\prime}$ de até $8.000^{x} \mathrm{psi}$.

CONCLUSÕES Neste trabalho, mostramos que a análise conjunta dos perfis $R G, D T$ e $r$ pode resultar no conhecimento de parâmetros de perfuração e na seleção de brocas, através do cálculo de $T_{x}, \mathrm{C}^{\prime}$ e $E_{S}$. Para atingir tais objetivos, foram utilizados dados dos Poços 1 e 2 do Golfo do México, cujos intervalos estudados indicaram melhores opções de brocas que aquelas que foram utilizadas na realidade. Desta forma, essa metodologia pode ser utilizada em futuras campanhas de perfuração de poços nos campos de petróleo sob estudo. Mas, é importante ressaltar que essa metodologia não substitui completamente a experiência dos profissionais envolvidos num projeto de perfuração. Como foi apresentado ao longo do artigo, muitas vezes é preferível perfurar a uma taxa mais lenta e evitar a troca de broca por desgaste, que furar de forma mais rápida e substituí-la continuamente, precisando, assim, içar toda a coluna de perfuração. Portanto, a interpretação dos dados dos perfis geofísicos de poços permite a otimização de um projeto e a montagem de uma estratégia de perfuração, o que se traduz numa melhor utilização dos recursos locados nessa atividade.

Agradecimentos Os autores agradecem à UENF pelo apoio computacional, à UFRJ, à BAKER HUGHES e à PETROBRAS pela participação dos autores ML, JS, LL, RC e SA. AC agradece ao CNPq pela bolsa de produtividade em pesquisa. Finalmente, agradecem aos engenheiros Daltro Carvalho, Ilmar Fruet e Raul Sato da PETROBRAS, pelas suas valiosas sugestões.

\section{Referências}

Asquith G. \& Gibson C. 1982. Basic well log analysis for geologists. AAPG, Oklahoma, $216 \mathrm{p}$.

Caicedo U., Calhoun M., Ewy T. 2005. Unique ROP prediction using bit-specific coefficient of sliding friction and mechanical efficiency as a function of confined compressive strength impacts drilling performance. In: SPE/ IADC Drilling Conference, Amsterdam, Netherlands. Paper number SPE 92576-MS. Disponível em www.onepetro.org/mslib/app/search.do, acesso em 15/01/2008.

Curry D., Fear, M., Govzitch A., Aghazada L. 2005. Technical limit specific energy - an index to facilitate drilling performance evaluation. In: SPE/IADC Drilling Conference, Amsterdam, Netherlands. Paper number 92318MS. Disponível em: www.onepetro.org/mslib/app/search.do, acesso em 15/01/2008.

Dagrain F. \& Tshibangu J.P. 2002. Use of the D3 model for the estimation of forces acting on a cutter in rock cutting and drilling. In: SPE/ISRM Rock Mechanical Conference, Irving, Texas. Paper number SPE 78242-MS. Disponível em: www.onepetro.org/mslib/app/search.do, acesso em 15/01/2008.

Dumans C.F.F. 1990. PDC bit selection method through the analysis of past bit performances. In: SPE Latin America Petroleum Engineering Conference, Rio de Janeiro, Brazil. Paper number SPE 21073. Disponível em: www.onepetro.org/mslib/app/search.do, acesso em 15/01/2008.

Dupriest F.E. \& Koederitz L. 2005. Maximizing drill rates with real-time surveillance of mechanical specific energy. In: SPE/IADC Drilling Conference, Amsterdam, Netherlands. Paper Number SPE 92194-MS. Disponível em: www.onepetro.org/mslib/app/search.do, acesso em 15/01/2008.
Farrelly M.E. \& Rabia H. 1987. Bit performance and selection: a novel approach. In: SPE/IADC Drilling Conference, New Orleans, Louisiana, Paper Number SPE 16163-MS. Disponível em: www.onepetro.org/mslib/ app/search.do, acesso em 15/01/2008.

Erling F., Rune M.H., Per H., Arne M.R. 1991. Petroleum related rock mechanics. Elsevier, Amsterdam, 338 p.

Farrelly M.E. \& Rabia H. 1987. Bit performance and selection: a novel approach. In: SPE/IADC Drilling Conference.

Gore R.H. 1992. The Gulf of Mexico. Pineapple Press, Inc. Sarasota Florida, 384 p.

Leite M.V.C. \& Lobo L.F. 2006. Estimativa da compressibilidade axial das rochas utilizando a técnica de lógica fuzzy e o conceito de energia específica: aplicação em poços do Campo de Namorado e do Golfo do México. Monografia de Aperfeiçoamento/Especialização em Curso de Especialização em Engenharia de Petróleo/PETROBRAS), Universidade Federal da Bahia, Salvador, $138 \mathrm{p}$.

Rabelo S. 2004. Estudo sobre a viabilidade do cálculo da porosidade com base em um conjunto reduzido de perfis e sua aplicação na Bacia de Almada/BA. Dissertação de Mestrado, Macaé, Universidade Estadual do Norte Fluminense Darcy Ribeiro/Laboratório de Engenharia e Exploração de Petróleo, 320 p.

Teale R. 1965. The concept of specific energy in rock drilling. Int. J. Rock Mech and Mining Sci, 2:57-73.

Manuscrito ID 10557

Submetido em 14 de fevereiro de 2008 Aceito em 26 de maio de 2011 